\title{
Relationship between deep venous thrombosis and inflammatory cytokines in postoperative patients with malignant abdominal tumors
}

\author{
T. Du and Z. Tan \\ Department of General Surgery, Zhongnan Hospital, School of Medicine, \\ National Wuhan University, Wuhan, Hubei Province, China
}

\begin{abstract}
Deep venous thrombosis (DVT) is a common surgical complication in cancer patients and evidence that inflammation plays a role in the occurrence of DVT is increasing. We studied a population of cancer patients with abdominal malignancies with the aim of investigating whether the levels of circulating inflammatory cytokines were associated with postoperative DVT, and to determine the levels in DVT diagnoses. The serum levels of C-reactive protein (CRP), interleukins (IL)-6 and IL-10, nuclear transcription factor- $\mathrm{KB}(\mathrm{NF}-\kappa \mathrm{B})$ and $\mathrm{E}$-selectin (E-Sel) were determined in 120 individuals, who were divided into 3 groups: healthy controls, patients with and patients without DVT after surgery for an abdominal malignancy. Data were analyzed by ANOVA, Dunnet's T3 test, chi-square test, and univariate and multivariate logistic regression as needed. The CRP, IL-6, NF$\kappa B$, and $\mathrm{E}$-Sel levels in patients with DVT were significantly higher than those in the other groups $(\mathrm{P}<0.05)$. The IL-10 level was higher in patients with DVT than in controls but lower than in patients without DVT. Univariate analysis revealed that CRP, IL-6, $\mathrm{NF}-\kappa \mathrm{B}$, and $\mathrm{E}-\mathrm{Sel}$ were statistically associated with the risk of DVT $(\mathrm{OR}=1.98, \mathrm{P}=0.002 ; \mathrm{OR}=1.17, \mathrm{P}=0.000 ; \mathrm{OR}=1.03$, $\mathrm{P}=0.042$; and $\mathrm{OR}=1.38, \mathrm{P}=0.003$; respectively), whereas $\mathrm{IL}-10$ had a protective effect $(\mathrm{OR}=0.94, \mathrm{P}=0.011)$. Multivariate analysis showed that $E$-Sel was an independent risk factor $(O R=1.41, P=0.000)$. Thus, this study indicated that an increased serum level of E-Sel was associated with increased DVT risk in postoperative patients with abdominal malignancy, indicating that E-Sel may be a useful predictor of diagnosis of DVT.
\end{abstract}

Key words: Malignant tumor; Abdominal surgery; Deep venous thrombosis (DVT); Inflammatory cytokine; Correlation

\section{Introduction}

The incidence of venous thromboembolism (deep venous thrombosis, DVT) and pulmonary embolism (PE) in cancer is gradually increasing. During the acute phase of DVT, 10 to $40 \%$ of patients will develop PE, with a mortality rate of 10 to $20 \%$ (1). According to Akl et al., both surgical removal of malignant tumors and abdominal surgery are high-risk factors for DVT (2). DVT and PE are common postoperative complications in cancer patients, and their treatment is accompanied by recurrence and bleeding complications. Thromboprophylaxis is thus important for prognosis and quality of life in this group of patients.

The mechanism of acute lower-limb DVT after surgical treatment of abdominal malignancies comprises several factors, including coagulation disorders, which are present in most tumor patients. The damage to vascular endothelial cells caused by abdominal surgery and tissue injury, coagulation disorders, and tissue injury are thus interrelated processes in thrombosis (3). Coagulation disorders can greatly affect the occurrence of acute thrombosis, and the role of inflammatory cytokines in coagulation disorders has been illustrated by various studies. As key factors in inflammatory reactions, mediators of inflammation are mutually amplified during thrombosis (4). Several clinical trials have established that elevated inflammatory cytokine levels play a relevant prothrombotic role in coronary atherosclerotic heart disease, cerebrovascular diseases and diabetic peripheral angiopathy (5). However, the main purpose of those studies was to explore the association between inflammatory cytokines and arterial diseases, and they seldom focused on the relationship between inflammatory cytokines and lower-limb DVT.

We enrolled cancer patients who had undergone abdominal surgery and compared the serum concentrations of several inflammatory cytokines in healthy normal individuals and in patients with or without lower-limb DVT

Correspondence: Z. Tan, Department of General Surgery, Zhongnan Hospital of Hubei Province, School of Medicine, National Wuhan University, 169 Donghu Road, Wuhan (430071), Hubei Province, China. E-mail: tanz728@hotmail.com and/or dt20006@hotmail.com 
after surgical removal of a malignant abdominal tumor. The purpose of this study was to determine whether inflammatory cytokine levels could be used to predict postoperative acute DVT in patients with malignant abdominal tumors. If so, they could be helpful for targeting prophylactic therapy to reduce the incidence of this serious complication.

\section{Patients and Methods}

\section{Study design and clinical data}

This case-control study was designed to explore the relationship between various inflammatory cytokines and postoperative acute DVT in patients with abdominal malignancy. The study was performed at the Department of Clinical Laboratories of Zhongnan Hospital, Wuhan, China, from July 2009 to December 2012 according to the ethical principles approved by the local Ethics Committee.

One hundred and twenty cases were divided into 3 groups. These included 40 normal, healthy volunteer controls recruited during routine physical examinations and 80 inpatients in the Departments of General Surgery, Urological Surgery or Gynecological Tumors. Forty inpatients were postoperative patients treated for abdominal malignancies and were without DVT. This group comprised 6 cases of gastric cancer, 8 of right colon cancer, 4 of hepatocellular carcinoma, 2 of pancreatic cancer, 10 of rectal cancer, 4 of oophoroma, 4 of carcinoma of the uterine cervix, and 2 of carcinoma of the prostate. Forty were inpatients with lower-limb DVT after surgical treatment of abdominal malignancies. This group comprised 4 cases of gastric cancer, 6 of right colon cancer, 6 of hepatocellular carcinoma, 2 of pancreatic cancer, 10 of rectal cancer, 6 of oophoroma, 4 of carcinoma of the uterine cervix, and 2 of carcinoma of the prostate.

Normal blood samples were obtained from healthy volunteers during routine physical examinations at the medical examination center. The inclusion criteria for volunteers were: physically healthy with normal blood sugar levels, hepatic and renal function, routine blood and urine tests and coagulation function. The exclusion criteria were long-term bed rest (e.g., following fracture or paralysis), a history of thrombosis, a long-term history of corticosteroid administration, and chronic liver disease. Postoperative patients with malignant abdominal tumors and a diagnosis established by pathological examination were eligible for inclusion. Patients with DVT were diagnosed by two-color duplex ultrasonography or ascending venography within 2 to 10 days after surgical treatment. The exclusion criteria were DVT diagnosed more than 10 days after surgery, an abnormal preoperative platelet count, peritonitis, and emergency abdominal surgery. The remaining exclusion criteria were similar to those for the physically healthy examinees.

\section{Reagents and protocol for the determination of inflammatory cytokine levels}

Enzyme-linked immunosorbent assay (ELISA) kits for human serum $\mathrm{C}$-reactive protein (CRP), interleukin-6 (IL-6) and IL-10 were purchased from Calvin Biological Technology Co., Ltd., China. ELISA kits for human serum nuclear transcription factor $-\kappa \mathrm{B}(\mathrm{NF}-\kappa \mathrm{B})$ and E-selectin (E-Sel) were purchased from Sunbio Company, Korea. Whole-blood samples were collected in the early morning after overnight fasting. The samples were stored for $15 \mathrm{~h}$ at room temperature and then centrifuged at $1000 \mathrm{~g}$ for $20 \mathrm{~min}$. The supernatant was then separated and stored at $-70^{\circ} \mathrm{C}$ until testing. Repeated freezing and thawing were avoided for all samples. For the patients without DVT after surgery for an abdominal malignancy, wholeblood samples were collected on the tenth day after surgery, and whole-blood samples from the patients with lower-limb DVT were collected in the morning after the diagnosis. The procedures for the measurement of CRP, IL-6, IL-10, NF- $\mathrm{BB}$, and E-Sel levels followed the instructions provided by the kit manufacturers.

\section{Statistical analysis}

Statistical analysis was performed using the SPSS software (version 13.0, SPSS Inc., USA). Age, body mass index (BMI), and cytokine serum concentrations are reported as means $\pm S D$. Gender and smoking status are reported as constituent ratio. Mean values were analyzed using one-way analysis of variance (ANOVA) and Dunnet's T3 test; categorical data were compared by the chi-square test. The limit of statistical significance was set at $\mathrm{P}<0.05$. The association of circulating inflammatory cytokines levels with the risk of DVT in postoperative patients with abdominal malignancy was first evaluated by univariate logistic regression analysis. Significant variables, together with gender and age, were included in a forward stepwise multivariate logistic regression analysis to determine the independent risk factors for DVT (method LR, $\mathrm{P}=0.05$ to enter, $\mathrm{P}=0.01$ to remove).

\section{Results}

The demographic characteristics of all of the individuals in our study, including age, gender, BMI and smoking status, are reported in Table 1 . There were no statistically significant differences among groups related to age $(F=0.73, P=0.484)$ or $B M I ~(F=1.91, P=0.152)$ distribution. No significant between-group differences were observed for gender $\left(\chi^{2}=1.07, \mathrm{P}=0.585\right)$ or smoking status $\left(\chi^{2}=2.46, \mathrm{P}=0.292\right)$.

The comparisons of serum concentrations of inflammatory cytokines are reported in Table 2. There were statistically significant differences among the three groups for CRP, IL-6, IL-10, NF- $\kappa B$, and $E-S e l$ levels $\left(F_{C R P}=993.58, P=0.000 ; \quad F_{I L-6}=196.18, \quad P=0.000\right.$; $\mathrm{F}_{\mathrm{IL}-10}=32.76, \mathrm{P}=0.000 ; \mathrm{F}_{\mathrm{NF}-\mathrm{kB}}=42.33, \mathrm{P}=0.000$; and 
Table 1. Demographic and baseline characteristics of patients with and without deep venous thrombosis (DVT) after surgery for an abdominal malignancy and healthy controls.

\begin{tabular}{lccc}
\hline Parameters & $\begin{array}{c}\text { Healthy } \\
\text { controls }\end{array}$ & $\begin{array}{c}\text { Patients with- } \\
\text { out DVT }\end{array}$ & $\begin{array}{c}\text { Patients } \\
\text { with DVT }\end{array}$ \\
\hline Age & $42.8 \pm 9.6$ & $45.8 \pm 11.0$ & $44.7 \pm 10.5$ \\
Gender, $\mathrm{n}(\%)$ & & & \\
$\quad$ Male & $20(50 \%)$ & $20(50 \%)$ & $24(60 \%)$ \\
$\quad$ Female & $20(50 \%)$ & $20(50 \%)$ & $16(40 \%)$ \\
Smoker, $\mathrm{n}(\%)$ & $16(40 \%)$ & $17(42.5 \%)$ & $18(45 \%)$ \\
BMl $\left(\mathrm{kg} / \mathrm{m}^{2}\right)$ & $24.4 \pm 2.2$ & $24.9 \pm 2.0$ & $23.8 \pm 2.5$ \\
\hline
\end{tabular}

Data are reported as means $\pm S D$ or as number and percent for 40 subjects per group. Mean values were analyzed by one-way ANOVA; categorical data were compared by the chi-square test. There were no statistically significant differences.

$\mathrm{F}_{\mathrm{CRP}}=464.77, \mathrm{P}=0.000$, respectively). The control group had significantly lower CRP, IL-6, IL-10, NF- $\mathrm{BB}$, and E-Sel serum levels than both patients with or without DVT after surgery for abdominal malignancy. Patients with DVT had significantly higher CRP, IL-6, NF- $\mathrm{B}$ B and E-Sel serum levels, but lower IL-10 levels, than patients without DVT.

The results of risk analysis are reported in Table 3 . Univariate logistic regression analysis indicated that CRP, IL-6, IL-10, NF- $\mathrm{B} B$ and E-Sel were statistically significant factors. CRP, IL-6, NF- $\kappa B$, and E-Sel were associated with increased risk of DVT. In contrast, IL-10 was correlated with decreased risk. However, multivariate logistic regression demonstrated that $E$-Sel $(P=0.000)$ was an independent risk factor, and IL-6 $(P=0.088)$ was an indeterminate independent risk factor, Nagelkerke $\mathrm{R}^{2}=0.937$.

\section{Discussion}

A previous study showed that inflammatory reactions are involved in the development of DVT in patients with malignant tumors (6). The occurrence of malignant tumors, the development of DVT, and inflammatory reactions are closely linked by cell-cell communication and interaction. One of the ways in which cells interact and communicate is via soluble mediators such as cytokines and other chemical factors (7). Inflammatory cytokines can be divided into two types: pro-inflammatory cytokines and anti-inflammatory cytokines (8). Pro-inflammatory cytokines include tumor necrosis factor- $\alpha$ (TNF- $\alpha$ ), $\mathrm{IL}-1 \beta$, IL-6, and IL-8, among others, and anti-inflammatory cytokines (so-called "protective cytokines") include IL-2, IL-4, IL-10, IL-13, transforming growth factor- $\beta$ (TGF- $\beta$ ), and colony-stimulating factor (CSF), among others. There is a "cytokine balance" between pro-inflammatory and anti-inflammatory cytokines (9). However, when the body encounters an attack, such as trauma, infection, a tumor or surgery, the levels of these two types of cytokines vary dramatically (10). It is believed that regional or appropriate inflammatory reactions enhance the defensive capabilities of the body, but the excessive production and release of pro-inflammatory cytokines induces an uncontrolled chain reaction, further increasing their levels and constituting a "second attack" on the organism (11). That is, once the production of a type of cytokine has been stimulated, it can induce the production of itself and other cytokines in great amounts, stimulating a biochemical cascade. The thrombotic cascade is one example of such biochemical cascade (12).

In the present study, sera were collected from patients with or without DVT after surgical treatment for abdominal malignancies and compared with those of healthy controls. We observed significantly higher levels of IL-6, CRP, $\mathrm{NF}-\kappa \mathrm{B}, \mathrm{E}-\mathrm{sel}$, and IL-10 in surgical cancer patients than in controls regardless of diagnosis of DVT. There were also significant differences between surgical cancer patients with DVT and without DVT. Consistent with previous studies, the inflammatory reactions were stronger in patients with DVT according to the higher serum levels of IL-6, CRP, NF- $\kappa B$, E-sel, and lower IL-10 levels.

$\mathrm{CRP}$ is an acute reactive protein in humans, and in addition to acting as a marker of inflammation, it can also participate in the inflammatory process (13). In 2006, it was discovered that CRP is an independent risk factor for

Table 2. Comparison of serum cytokines in patients with and without deep venous thrombosis (DVT) after surgery for an abdominal malignancy and healthy controls.

\begin{tabular}{lccrrr}
\hline Factors & Healthy controls & Patients without DVT & Patients with DVT & $F$ & $P$ \\
\hline CRP $(\mathrm{mg} / \mathrm{L})$ & $2.51 \pm 0.37$ & $10.91 \pm 0.90^{+}$ & $11.20 \pm 1.22^{+*}$ & 993.58 & 0.000 \\
IL-6 $(\mathrm{pg} / \mathrm{mL})$ & $105.76 \pm 8.2$ & $130.81 \pm 9.38^{+}$ & $152.88 \pm 12.36^{+*}$ & 196.18 & 0.000 \\
IL-10 $(\mathrm{ng} / \mathrm{mL})$ & $30.83 \pm 3.22$ & $46.43 \pm 8.34^{+}$ & $40.72 \pm 10.56^{+*}$ & 32.76 & 0.000 \\
NF-kB $(\mu \mathrm{g} / \mathrm{L})$ & $50 \pm 13$ & $68 \pm 16^{+}$ & $85 \pm 20^{+*}$ & 42.33 & 0.000 \\
E-Sel $(\mu \mathrm{g} / \mathrm{mL})$ & $52 \pm 7$ & $82 \pm 6^{+}$ & $120 \pm 18^{+*}$ & 464.77 & 0.000 \\
\hline
\end{tabular}

Data are reported as means \pm SD for 40 subjects per group. Comparisons among groups were performed using one-way ANOVA. ${ }^{+} \mathrm{P}<0.05$ compared to healthy controls; ${ }^{*} \mathrm{P}<0.05$ compared to patients without DVT (Dunnet's T3 test). CRP: C-reactive protein; IL:

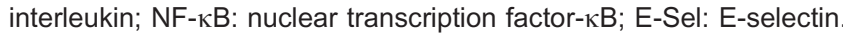


Table 3. Univariate and multivariate logistic regression analyses.

\begin{tabular}{|c|c|c|c|c|}
\hline \multirow[t]{2}{*}{ Factors } & \multicolumn{2}{|c|}{ Univariate logistic regression } & \multicolumn{2}{|c|}{ Multivariate logistic regression } \\
\hline & OR $(95 \% \mathrm{Cl})$ & $\mathrm{P}$ & OR $(95 \% \mathrm{Cl})$ & $\mathrm{P}$ \\
\hline Age & $0.99(0.96-1.03)$ & 0.660 & - & - \\
\hline Gender & $0.67(0.28-1.62)$ & 0.370 & - & - \\
\hline Smoker & $1.11(0.46-2.68)$ & 0.822 & - & - \\
\hline BMI & $0.88(0.72-1.08)$ & 0.208 & - & - \\
\hline CRP & $1.98(1.28-3.05)$ & 0.002 & - & - \\
\hline IL-6 & $1.17(1.10-1.27)$ & 0.000 & $1.15(0.98-1.36)$ & 0.088 \\
\hline IL-10 & $0.94(0.90-0.99)$ & 0.011 & - & - \\
\hline$N F-\kappa B$ & $1.03(1.01-1.06)$ & 0.042 & - & - \\
\hline E-sel & $1.38(1.00-1.72)$ & 0.003 & $1.41(1.05-1.90)$ & 0.000 \\
\hline
\end{tabular}

Analyses were performed between patients with and without deep venous thrombosis after surgery for an abdominal malignancy. BMI:

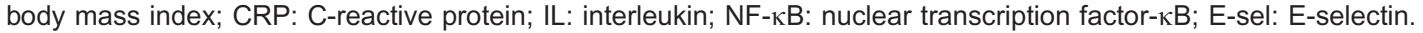

coronary atherosclerotic heart disease in patients with diabetes (14). A scientific team led by Dr. Mark B. Pepys announced that their new compound (1,6-bis(phosphocholine)-hexane) could inhibit the damage to an organism caused by CRP, and as a result could reduce the risk of cardiac events, and myocardial infarction, in particular (15). IL- 6 can be synthesized and secreted by various cells, such as activated $T$ lymphocytes and B lymphocytes, and, similar to CRP, it is an important cytokine in acute episodes of inflammation. IL- 6 can induce NF- $\kappa B$ expression and then activate the polarized expression of intercellular adhesion molecule-1 (ICAM-1) (16). This cytokine can also increase the ability of platelets to respond to thrombin and then promote the development of thrombosis (17). It is interesting that IL-6 was independent but was not statistically significant $(P=0.088)$; we may need to enlarge the sample size to do further research on this. NF- $\kappa B$ is a key factor regulating gene transcription and is involved not only in the process of inflammation but also in the development of DVT, in which it plays a very important role (18).

IL-10 is an anti-inflammatory cytokine with multiple functions, including both anti-inflammatory activity and the ability to restrain immune reactions. A study by Proctor et al. (19) indicated that IL-10 could be an important clinical predictor of thrombosis. IL-10 has also been found to exert anticoagulant properties (20). It was also reported that IL-10 could reduce vein wall inflammation in an animal model and had the ability to protect endothelial cells (21). The serum level of IL-10 in DVT patients after the surgical treatment of abdominal malignancies was significantly lower than in those without postoperative DVT. It is likely that increased concentrations of IL-10 reduce the possibility of a cascade reaction induced by inflammatory mediators, and to a certain extent prevent the development of the thrombotic cascade. The results also demonstrated that IL-10 could be a target for the prevention and treatment of postoperative DVT following the surgical treatment of abdominal malignancies.

During the inflammation process most leukocytes require E-Sel as an acceptor to make contact with endothelial cells. E-sel has been reported to augment the thrombotic response (22) and to promote inflammatory reactions and stimulation of the thrombotic cascade (23). ESel polymorphism has also been correlated with tissue factor-mediated coagulation (24). The regulation of E-Sel expression on the cell surface is thus important for leukocyte adherence and extravasation.

This study has a few limitations. Different types of surgery, tumor site, and stage of the neoplasia may cause various patterns of cytokine expression. Several factors would interfere the levels of circulating cytokines, and we cannot ignore latent ones. Only thrombosis occurring within 10 days following surgery was recorded; tardus thromboses were not considered. In addition, a larger matched cohort follow-up study would effectively minimize the confounding factors, yet we also need to quantify the correlation and find a cut-off value to discriminate highfrom low-risk patients.

In conclusion, we observed that an elevated E-sel serum level was associated with higher DVT risk in surgical cancer patients, providing future prospects for clinical prediction of a DVT diagnosis.

\section{Acknowledgments}

We thank the Department of Urological Surgery and the Department of Gynecological Tumors of Zhongnan Hospital for providing the patients. We are grateful to the Wuhan City Science and Technology Bureau, Hubei Province, China, for funding the end of the study (No. HF-2010-103). 


\section{References}

1. Stein PD, Goodman LR, Hull RD, Dalen JE, Matta F. Diagnosis and management of isolated subsegmental pulmonary embolism: review and assessment of the options. Clin Appl Thromb Hemost 2012; 18: 20-26, doi: 10.1177/1076029611422363.

2. Akl EA, Vasireddi SR, Gunukula S, Yosuico VE, Barba M, Sperati $F$, et al. Anticoagulation for patients with cancer and central venous catheters. Cochrane Database Syst Rev 2011; CD006468.

3. Tufano A, Coppola A, Cerbone AM, Ruosi C, Franchini M. Preventing postsurgical venous thromboembolism: pharmacological approaches. Semin Thromb Hemost 2011; 37: 252-266, doi: 10.1055/s-0031-1273089.

4. Sood V, Luke CE, Deatrick KB, Baldwin J, Miller EM, Elfline $\mathrm{M}$, et al. Urokinase plasminogen activator independent early experimental thrombus resolution: MMP2 as an alternative mechanism. Thromb Haemost 2010; 104: 1174-1183, doi: 10.1160/TH10-03-0184.

5. Xenos ES, Vargas HD, Davenport DL. Association of blood transfusion and venous thromboembolism after colorectal cancer resection. Thromb Res 2012; 129: 568-572, doi: 10.1016/j.thromres.2011.07.047.

6. van den Berg YW, Versteeg HH. Alternatively spliced tissue factor. A crippled protein in coagulation or a key player in non-haemostatic processes? Hamostaseologie 2010; 30: 144-149.

7. Rudolph V, Freeman BA. Cardiovascular consequences when nitric oxide and lipid signaling converge. Circ Res 2009; 105: 511-522, doi: 10.1161/CIRCRESAHA.109. 202077.

8. New SE, Aikawa E. Cardiovascular calcification: an inflammatory disease. Circ J 2011; 75: 1305-1313, doi: 10. 1253/circj.CJ-11-0395.

9. Marik PE, Flemmer M. The immune response to surgery and trauma: Implications for treatment. J Trauma Acute Care Surg 2012; 73: 801-808, doi: 10.1097/TA.0b013e $318265 \mathrm{cf} 87$

10. Chen W, Li Z, Bai L, Lin Y. NF-kappaB in lung cancer, a carcinogenesis mediator and a prevention and therapy target. Front Biosci 2011; 16: 1172-1185, doi: 10.2741/ 3782.

11. Burton-Freeman B. Postprandial metabolic events and fruitderived phenolics: a review of the science. Br J Nutr 2010; 104 (Suppl 3): S1-S14, doi: 10.1017/S0007114510003909.

12. Frangogiannis NG. The immune system and cardiac repair. Pharmacol Res 2008; 58: 88-111, doi: 10.1016/j.phrs. 2008.06.007.
13. Kones R. Rosuvastatin, inflammation, C-reactive protein JUPITER, and primary prevention of cardiovascular disease - a perspective. Drug Des Devel Ther 2010; 4: 383-413, doi: 10.2147/DDDT.S10812.

14. Shah R, Burg MM, Vashist A, Collins D, Liu J, Jadbabaie F, et al. C-reactive protein and vulnerability to mental stressinduced myocardial ischemia. Mol Med 2006; 12: 269-274 doi: 10.2119/2006-00077. Shah.

15. Pepys MB, Hirschfield GM, Tennent GA, Gallimore JR, Kahan MC, Bellotti V, et al. Targeting C-reactive protein for the treatment of cardiovascular disease. Nature 2006; 440 1217-1221, doi: 10.1038/nature04672.

16. Medzhitov R, Shevach EM, Trinchieri G, Mellor AL, Munn $\mathrm{DH}$, Gordon S, et al. Highlights of 10 years of immunology in Nature Reviews Immunology. Nat Rev Immunol 2011; 11 : 693-702, doi: 10.1038/nri3063.

17. Obitsu $Y$, Shigematsu H. [Deep vein thrombosis in patients with cancer]. Gan To Kagaku Ryoho 2009; 36: 535-539.

18. Boon RA, Horrevoets AJ. Key transcriptional regulators of the vasoprotective effects of shear stress. Hamostaseologie 2009; 29: 39-43.

19. Proctor MC, Sullivan $V$, Zajkowski P, Wolk SW, Pomerantz RA, Wakefield TW, et al. A role for interleukin-10 in the assessment of venous thromboembolism risk in injured patients. J Trauma 2006; 60: 147-151, doi: 10.1097/01.ta. 0000197180.79965.bc

20. Ben-Hadj-Khalifa S, Nguyen $P$, Mahjoub $T$, Hezard $N$. Anticoagulant properties of the anti-inflammatory cytokine IL-10 in a factor Xa-activated human monocyte model. Eur Cytokine Netw 2012; 23: 87-92.

21. Ahlers O, Nachtigall I, Lenze J, Goldmann A, Schulte E, Hohne $\mathrm{C}$, et al. Intraoperative thoracic epidural anaesthesia attenuates stress-induced immunosuppression in patients undergoing major abdominal surgery. $\mathrm{Br} J$ Anaesth 2008 101: 781-787, doi: 10.1093/bja/aen287.

22. Myers D Jr, Farris D, Hawley A, Wrobleski S, Chapman A Stoolman $\mathrm{L}$, et al. Selectins influence thrombosis in a mouse model of experimental deep venous thrombosis. J Surg Res 2002; 108: 212-221, doi: 10.1006/jsre.2002.6552.

23. Zakynthinos E, Pappa N. Inflammatory biomarkers in coronary artery disease. J Cardiol 2009; 53: 317-333, doi: 10.1016/j.jjcc.2008.12.007.

24. Yoshida M, Takano Y, Sasaoka T, Izumi T, Kimura A. Eselectin polymorphism associated with myocardial infarction causes enhanced leukocyte-endothelial interactions under flow conditions. Arterioscler Thromb Vasc Biol 2003; 23 783-788, doi: 10.1161/01.ATV.0000067427.40133.59. 